\title{
74-year-old man with left main and carotid artery disease - how life can change plans
}

\author{
Piotr Kübler', Dawid Ilnicki², Artur Telichowski', Krzysztof Reczuch ${ }^{1,3}$ \\ ${ }^{1}$ Cardiology Department, Military Hospital of Wroclaw, Poland \\ 2Students Scientific Association, Department of Heart Diseases, Medical University of Wroclaw, Poland \\ 3Department of Heart Disease, Medical University of Wroclaw, Poland
}

Postep Kardiol Inter 2013; 9, 3 (33): 246-249

DOI: $10.5114 /$ pwki.2013.37503

\begin{abstract}
An unexpected incident or rapid deterioration of a patient's condition may require optimal adaptation of the treatment to the current state of the patient. We present a case of a 74-year-old man with significant left main coronary artery stenosis and tight stenoses of both carotid arteries. The case was initially qualified for bypass grafting with accompanying carotid artery endarterectomy, but an unexpected accident changed our way of treatment. Three days after angiography the patient suffered an ischemic stroke. We held a multidisciplinary meeting of the "Neuro-Vascular-Heart Team" and decided to treat the patient percutaneously.
\end{abstract}

Key words: carotid artery disease, left main disease.

\section{Introduction}

Sometimes an unexpected incident or rapid deterioration of a patient's condition requires a radical change in the prearranged way of treatment. In such cases it is essential to optimally adapt the treatment to the current state of the patient.

We present a case of a 74-year-old man with multivessel coronary disease and stenoses of carotid arteries, initially qualified for coronary artery bypass grafting (CABG) with simultaneous carotid endarterectomy (CEA), who experienced a recent ischemic stroke during hospitalization. This incident forced a change of the treatment plans of the patient.

\section{Case report}

A 74-year-old man with a history of ischemic heart disease, after 2 procedures of percutaneous coronary intervention $(\mathrm{PCl})$ of the right coronary artery ( $\mathrm{RCA}$ ) with baremetal stent implantation (in 2007 and 2008) and after balloon angioplasty because of restenosis in 2010, with arterial hypertension, chronic (most likely postinflammatory) kidney disease (CKD) in stage 3 according to the KDOQI (Kidney Disease Outcomes Quality Initiative), was admitted to our center in order to perform elective coronary angiography.

In anamnesis the patient reported a recurrence of typical angina in CCS class III/IV in recent months (severe chest pain during everyday activities). During physical examination a loud murmur over both carotid arteries drew our attention. Laboratory tests revealed an elevated level of serum creatinine $(1.63 \mathrm{mg} / \mathrm{dl})$ with estimated glomerular filtration rate (GFR) $41 \mathrm{ml} / \mathrm{min}$. Echocardiography showed normal left ventricular size, without regional contractility abnormalities, with normal left ventricular ejection function ( $(\mathrm{VEF}=60 \%$ ) and residual mitral and tricuspid regurgitation. Moreover, significant stenoses of both internal carotid arteries in echo-Doppler were found.

Coronary angiography demonstrated multivessel disease with significant stenosis of the left main coronary artery (LMCA) in the medial and distal part to $60-70 \%$, non-significant changes in the left anterior descending artery, diagonal branch and circumflex artery (Figure 1) and angiographically significant stenosis of RCA in the distal part about 70-80\% (Figure 2). Simultaneously performed carotid angiography demonstrated bilateral, very tight stenoses of both right (Figure 3) and left (Figure 4) internal carotid arteries, angiographically even tighter on the left side. Taking into account the overall clinical picture with intermediate values of Euro-Score (8\%) and Syntax-Score (28) the patient was initially qualified for CABG surgery with the suggestion of simultaneous carotid endarterectomy.

Meanwhile, in the morning of the third day of hospitalization the patient suddenly lost consciousness and suf-

\section{Corresponding author:}

Piotr Kübler MD, Cardiology Department, Military Hospital, 5 Weigla St, 50-981 Wroclaw, Poland, tel/fax: +48 717660250 ,

e-mail: pkubler75@gmail.com

Received: 5.02.2013, accepted: 8.08.2013. 


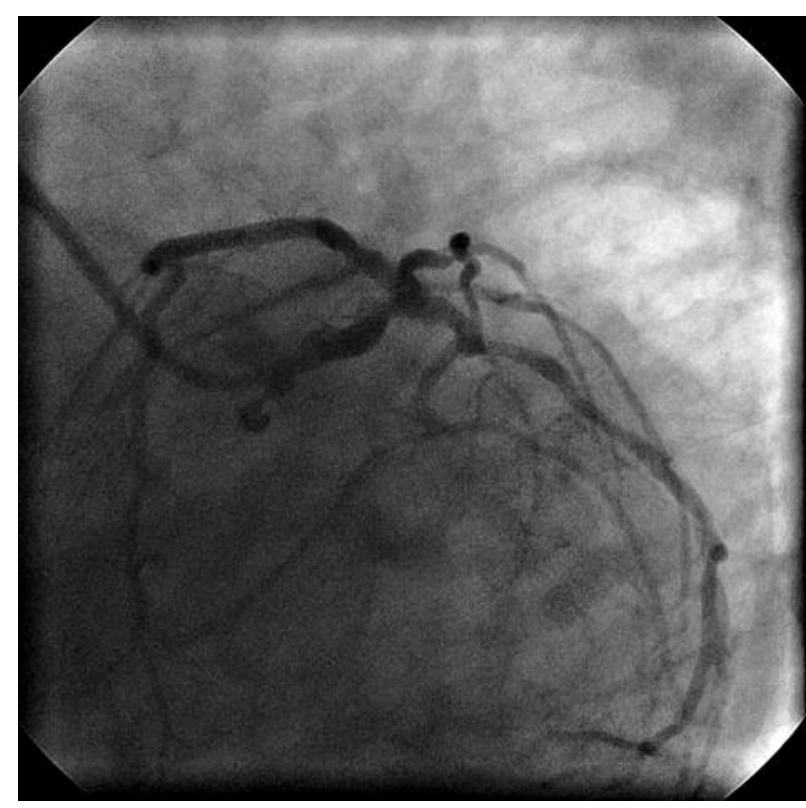

Fig. 1. Left coronary artery with significant left main coronary artery stenosis

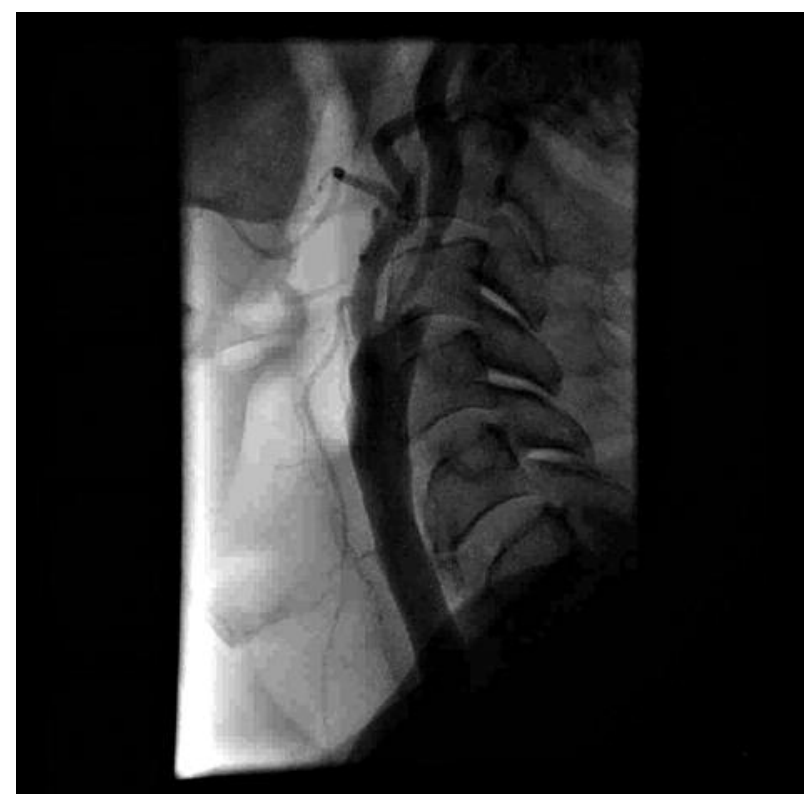

Fig. 3. Tight stenosis of right internal carotid artery

fered a head injury, but without accompanying chest pain or arrhythmia. He was hemodynamically stable, but a discreet hemiparesis of the right limbs was diagnosed. After head computed tomography and magnetic resonance imaging a diagnosis of recent ischemic stroke of the left hemisphere was confirmed.

Due to this unexpected incident we decided to hold an interdisciplinary meeting and to answer the question: how should we optimally treat our patient? The team was called the "Neuro-Vascular-Heart Team" and consisted of a neu-

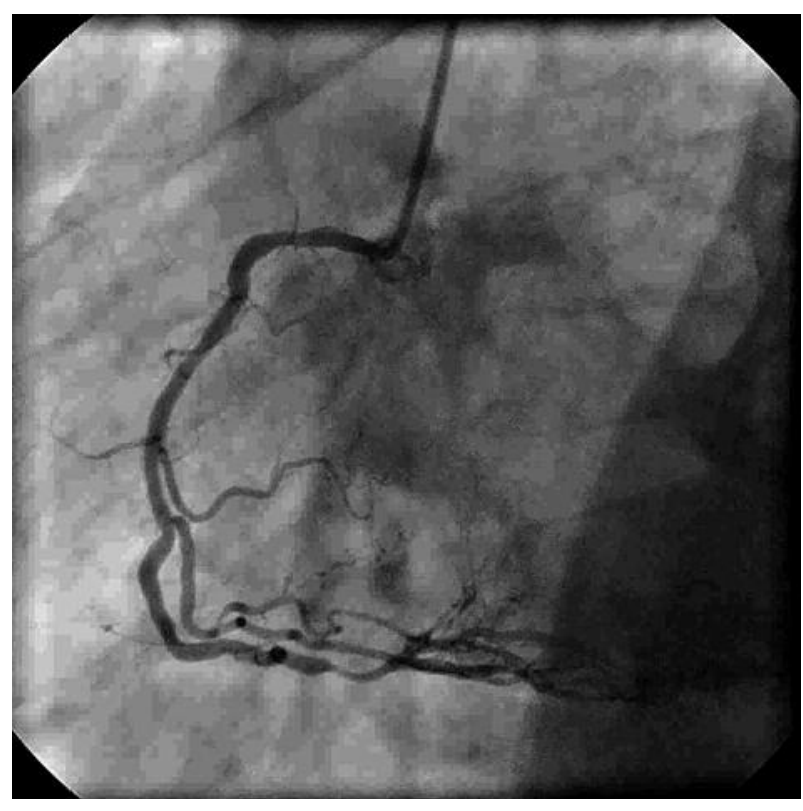

Fig. 2. Right coronary artery with distal narrowings

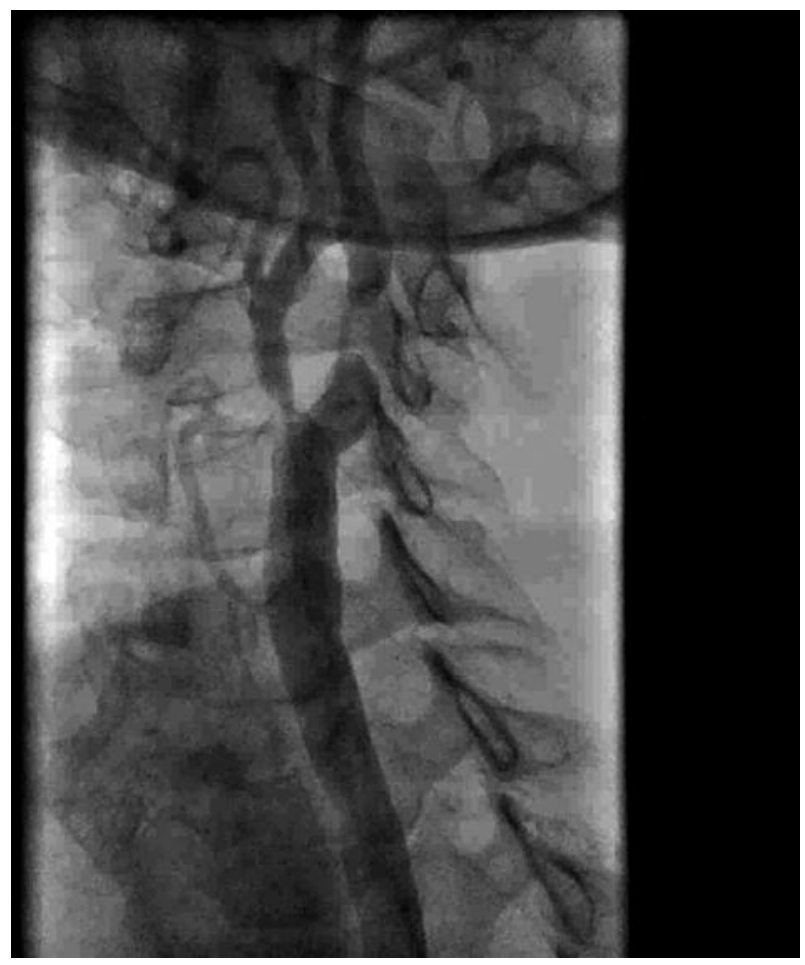

Fig. 4. Subtotal stenosis of left internal carotid artery

rologist, vascular and cardiac surgeon, interventional cardiologist and general cardiologist caring for the patient. However, proposals for further treatment were quite inconclusive and establishing a common position proved extremely difficult. From the perspective of the neurologist conservative treatment should be continued with optimal phar- 
macological treatment and careful monitoring, because the risk of any intervention in the current situation was too high. The surgeons' approach was obviously not conservative from their point of view the patient should undergo CABG, with prior CEA, but after 4-6 weeks. Until then, any surgery is associated with an increased risk related to the period of stroke healing. But the position of the cardiologists was more aggressive: interventional treatment should not wait, and carotid artery revascularization should be performed within 2 weeks after the stroke. Moreover, we should not postpone the revascularization of highly symptomatic, tight LMCA stenosis, because we expose the patient to subsequent cardio-cerebrovascular events. The discussion was vigorous and all participants defended their position, so the consensus was not clear. Finally, although not unanimously, the team decided to treat the patient percutaneously.

Another difficult question was: what should the optimal percutaneous strategy be? Which first: $\mathrm{PCl}$, carotid artery stenting (CAS) or maybe both simultaneously? PCI of the LMCA only or PCI of the RCA at the same time? Unilateral or bilateral CAS? Our final strategy was as follows: after adequate hydration on the $7^{\text {th }}$ day after admission, effective CAS of the left internal carotid artery (LICA), which was the symptomatic artery, was performed, with a self-expandable stent and with the use of a vascular filter as a distal protection (Figure 5). On the $12^{\text {th }}$ day of hospitalization, after creatinine control and hydration, $\mathrm{PCl}$ of the LMCA with 2 drug-eluting stents was performed (Figure 6). There were no complications after both procedures, without renal function deterioration. After 14 days of hospital stay the patient was discharged home in stable condition. We recommended optimal medical therapy and possible subsequent steps of revascularization. Three months later $\mathrm{PCI}$ of the RCA was performed, because of ongoing exercise angina, and after 5 months CAS of the right internal carotid artery (RICA), because of neurological symptoms.

\section{Discussion}

Current guidelines of cardiac societies, as well as clinical experience, affected therapeutic decision-making in the analyzed case.

Large randomized clinical trials of myocardial revascularization [1] and the guidelines of the European Society of Cardiology (ESC) [2] show the advantage of CABG over PCI in patients with distal LMCA stenosis with 2-vessel disease (for CABG - class IA; for PCI - class IIb B). In stable ischemic heart disease American guidelines also prefer CABG in LMCA stenosis (class IB) and $\mathrm{PCl}$ can be considered as an alternative to surgery - in class Ila B or IIb B, depending on the operative risk associated with the anatomy of the coronary arteries and clinical condition, which is estimated on the basis of the appropriate scales [3]. Prescheduled CABG in the discussed case was an appropriate method of revascularization; however, the event of acute stroke forced a change in the management - from a surgical to a percutaneous strategy.

In patients with coronary artery disease atherosclerosis often affects other areas, including the carotid arteries. The CEA in such patients is the method of choice (class IB) [2]. However, according to European guidelines in patients with symptomatic (transient ischemic attack or stroke within the last 6 months) internal carotid artery stenosis greater than $70 \%$ it is recommended to undergo carotid revascularization within 2 weeks after the brain incident (class IB).

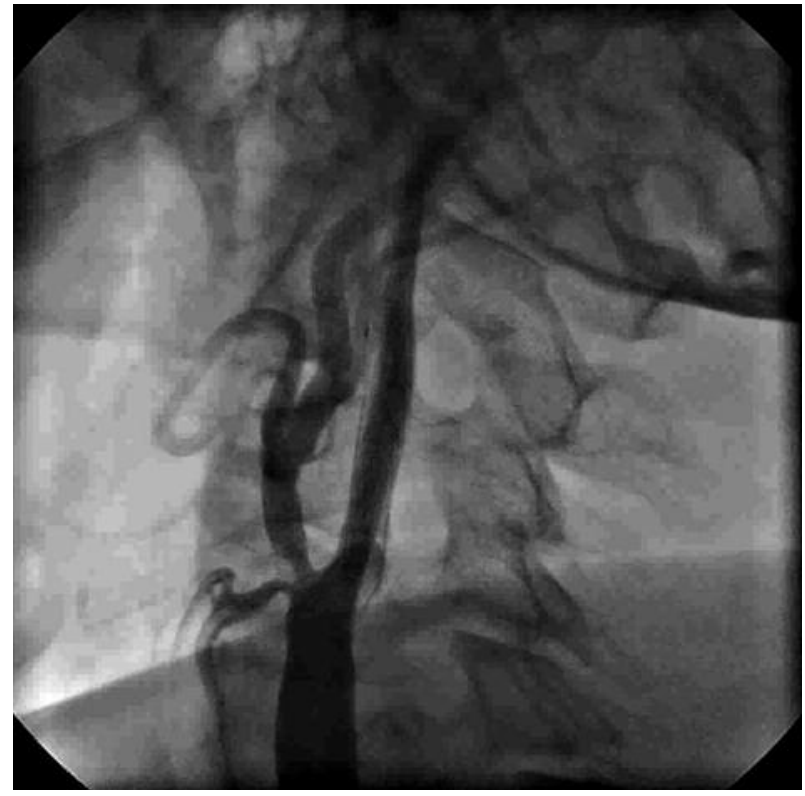

Fig. 5. Final result of left internal carotid artery stenting

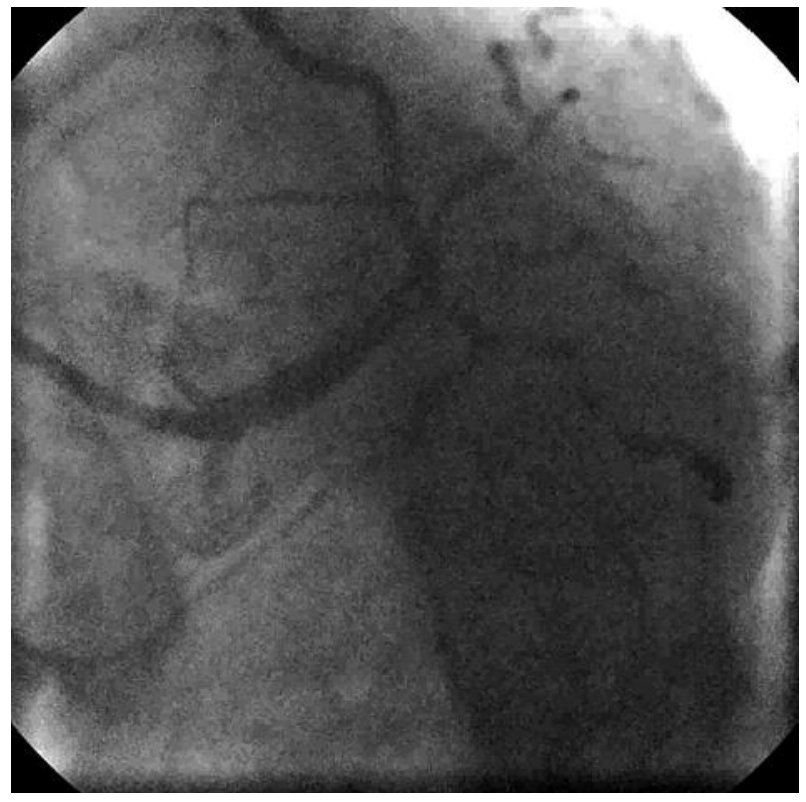

Fig. 6. Final result of left main coronary artery stenting 
In symptomatic patients with high surgical risk who require revascularization, CAS should be considered as an alternative for CEA (class Ila B) [4]. In contrast to the European guidelines, the American guidelines put in one line CEA and CAS, with a class of recommendation I for both surgical and percutaneous treatment (difference of strength of evidence: IA for CEA, IB for CAS) [5]. These guidelines emphasize our decision of prompt performance of CAS and not to risk further neurovascular incidents.

As recommended by the ESC, $\mathrm{PCl}$ and CAS should not be combined during the same procedure, except for simultaneous severe acute coronary and carotid syndromes (class IIIC) [2]. The order of revascularization during qualification for carotid and coronary artery revascularization depends on the clinical presentation - the first intervention should be performed on the more symptomatic vascular area (class IC). Finally, the decision to treat the patient percutaneously - initially CAS of the LICA and then $\mathrm{PCl}$ of the LMCA - clinically proved to be a successful choice.

And furthermore, ESC guidelines indicate CABG as a preferred method of coronary revascularization in patients with mild or moderate CKD (class Ila). In order to prevent acute kidney injury in these patients, surgery without the use of cardiopulmonary bypass may be considered [2]. In patients with CKD undergoing diagnostic tests or interventional treatment with the use of contrast agents, hydration of a patient with isotonic sodium chloride solution is recommended (class IA), for the prevention of contrast-induced nephropathy. In addition, in patients with mild, moderate or severe CKD, low osmolar or iso-osmolar contrast media should be used in a total dose of $<350 \mathrm{ml}$ or $<4 \mathrm{ml} / \mathrm{kg}$ (class IA) [2]. Intensive hydration and use of iso-osmolar contrast media proved to be sufficient in avoiding deterioration of renal function in the described patient.

\section{Conclusions}

This case shows that the treatment should be individualized, optimally adapted to the current condition of the patient. Guidelines are a signpost for us in making decisions, but they do not answer all the questions which arise when varied and complicated clinical situations occur. Concurrently, the international medical societies place emphasis on joint consultations and joint qualifications, to choose the optimal strategy for an individual patient, by interdisciplinary teams. This multidisciplinary team should collectively decide how to deal with the most difficult cases. Such an idea seems to be very attractive; however, in some situations the consensus may be difficult or even impossible to achieve and making bold decisions may be the most appropriate solution.

\section{References}

1. Serruys PW, Morice MC, Kappetein AP, et al.; SYNTAX Investigators. Percutaneous coronary intervention versus coronary-artery bypass grafting for severe CAD. N Engl J Med 2009; 360: 961-972.
2. Wijns W, Kolh P, Danchin N, et al. Guidelines on myocardial revascularization. The Task Force on Myocardial Revascularization of the European Society of Cardiology (ESC) and the European Association for Cardio-Thoracic Surgery (EACTS) and the European Association for Percutaneous Cardiovascular Interventions (EAPCI). Eur Heart J 2010; 31: 2501-2555.

3. Levine GN, Bates ER, Blankenship JC, et al. 2011 ACCF/AHA/SCAI Guideline for Percutaneous Coronary Intervention.A Report of the American College of Cardiology Foundation/American Heart Association Task Force on Practice Guidelines and the Society for Cardiovascular Angiography and Interventions. J Am Coll Cardiol 2011; 58: e44-e122.

4. Tendera M, Aboyans V, Bartelink ML, et al. ESC Guidelines on the diagnosis and treatment of peripheral artery diseases. Document covering atherosclerotic disease of extracranial carotid and vertebral, mesenteric, renal, upper and lower extremity arteries: The Task Force on the Diagnosis and Treatment of Peripheral Artery Diseases of the European Society of Cardiology (ESC). Endorsed by: the European Stroke Organisation (ESO). Eur Heart J 2011; 32: 2851-2906.

5. Brott TG, Halperin JL, Abbara S, et al. 2011 ASA/ACCF/AHA/AANN/ AANS/ACR/ASNR/CNS/SAIP/SCAI/SIR/SNIS/SVM/SVS guideline on the management of patients with extracranial carotid and vertebral artery disease: executive summary. J Am Coll Cardiol 2011; 57: e16-e94. 